\title{
Quantum Well Intermixing and Radiation Effects in InGaN/GaN Multi Quantum Wells
}

\author{
K. Lorenz ${ }^{* a}$, A. Redondo-Cubero, ${ }^{\mathrm{a}, \mathrm{b}}$, M. B. Lourenço ${ }^{\mathrm{a}}$, M. C. Sequeira ${ }^{\mathrm{a}}$, M. Peres ${ }^{\mathrm{a}}$, A. Freitas ${ }^{\mathrm{a}}$,

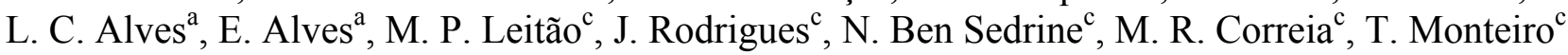 \\ ${ }^{a}$ Instituto Superior Técnico, Campus Tecnológico e Nuclear, Estrada Nacional 10, P-2695-066 \\ Bobadela LRS, Portugal; ${ }^{\mathrm{b}}$ Departamento de Física Aplicada y Centro de Micro-Análisis de \\ Materiales, Universidad Autónoma de Madrid, Madrid, Spain; ' ${ }^{\circ}$ epartamento de Física e i3N, \\ Universidade de Aveiro, 3810-193 Portugal
}

\begin{abstract}
Compositional grading of InGaN/GaN multi quantum wells (QWs) was proposed to mitigate polarization effects and Auger losses in InGaN-based light emitting diodes [K. P. O'Donnell et al., Phys. Status Solidi RRL 6 (2012) 49]. In this paper we are reviewing our recent attempts on achieving such gradient via quantum well intermixing. Annealing up to $1250{ }^{\circ} \mathrm{C}$ resulted in negligible interdiffusion of QWs and barriers revealing a surprising thermal stability well above the typical MOCVD growth temperatures. For annealing at $1400^{\circ} \mathrm{C}$ results suggest a decomposition of the QWs in regions with high and low InN content. The defect formation upon nitrogen implantation was studied in detail. Despite strong dynamic annealing effects, which keep structural damage low, the created defects strongly quench the QW luminescence even for low implantation fluences. This degradation could not be reversed during thermal annealing and is hampering the use of implantation induced quantum well intermixing in InGaN/GaN structures.
\end{abstract}

Keywords: InGaN/GaN, quantum wells, quantum well intermixing, ion implantation

\section{INTRODUCTION}

Polarization effects and Auger losses in $\mathrm{InGaN} / \mathrm{GaN}$ quantum well (QW) structures are considered among the main reasons for the low efficiency of nitride LEDs emitting at long wavelengths (green and beyond) as well as for the strong efficiency droop at high current densities ${ }^{1,2}$. The polarization induced electric fields cause a spatial separation of the electron and hole wave functions in the QW reducing strongly the radiative recombination rate (the Quantum Confined Stark Effect, QCSE) ${ }^{3}$. Compositional grading of QWs can be used to tune the potential profile and strain distribution in a multi QW (MQW) in order to control the electron-hole recombination. In addition, the softening of heterostructure interfaces in the active region has been shown to eliminate Auger losses in quantum $\operatorname{dots}^{4}$. Simulations of green InGaN diodes, using a multiscale formalism showed the great potential of compositional grading in mitigating both QCSE and Auger effect ${ }^{5,6}$. In particular, a parabolic gradient was shown to increase the overlap of electron and hole wave functions within the QWs resulting in increased internal quantum efficiency as well as lower Auger losses.

Such a parabolic compositional grading inside the QWs can be realized by post-growth interdiffusion of QW and barrier materials. QW intermixing (QWI) can be achieved either by simple thermal annealing with or without the presence of impurities $^{7}$, or by laser irradiation ${ }^{8}$ or ion implantation ${ }^{9}$ followed by annealing. The latter case is schematically shown in Fig. 1. Starting with a conventional rectangular quantum well the introduction of point defects and breaking of the bonds by ion implantation facilitate diffusion during post-implant annealing. The annealing step then promotes well-barrier interdiffusion and removes the implantation damage. This approach has the advantage of facilitating lateral patterning and monolithic integration. QWI was used successfully in arsenide and phosphide based materials to tune the band gap of QW structures ${ }^{7-9}$.

*Lorenz@ctn.tecnico.ulisboa.pt; phone+351-219946052 


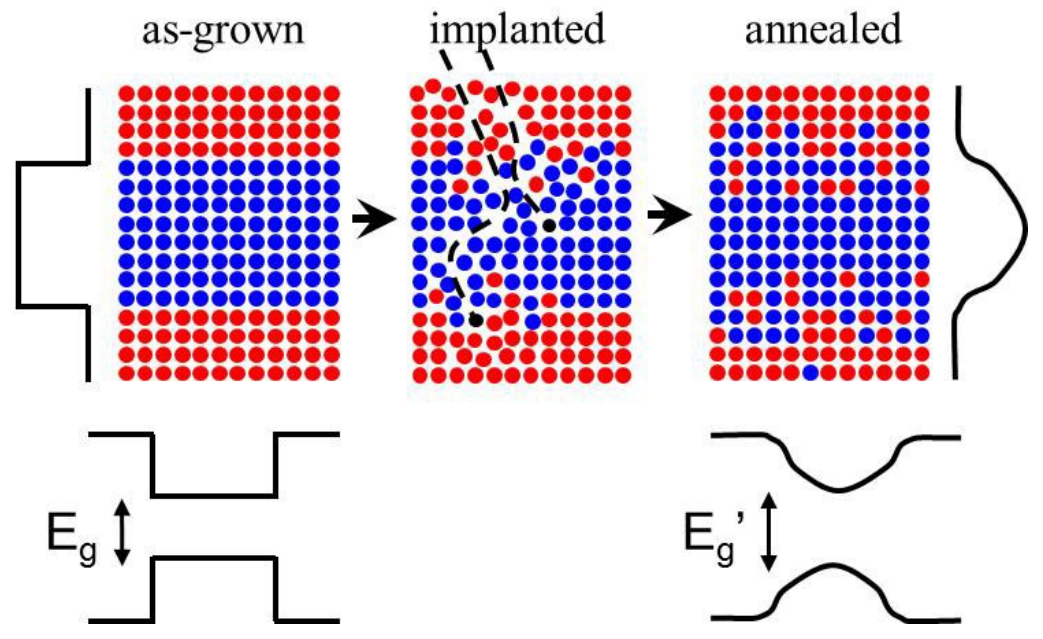

Figure 1. Schematics of the implantation induced QWI process. A QW with a rectangular composition profile is implanted. The created point defects promote interdiffusion of the elements in the QW and the barrier leading to a parabolic-like compositional gradient. Not taking into account any polarization effects the confinement potential is changed from rectangular to parabolic-like.

In the case of polar materials such as $\mathrm{GaN}$ and $\mathrm{ZnO}$ based QWs the situation is more complicated since the polarization effects distort the band structure leading to a triangular shape for a homogeneous composition and a parabolic-like shape after intermixing ${ }^{10}$. Recent work by Coleman et al. and Davis et al. showed the potential of ion-beam assisted QWI in wurtzite $\mathrm{ZnO} / \mathrm{ZnMgO} \mathrm{MQW}$ to increase the overlap of electron and hole wavefunctions and improve the internal quantum efficiency ${ }^{11,12}$. These authors showed that implantation can significantly enhance the intermixing process. In nitrides this subject has not been studied in detail yet although tunable emission upon intermixing of InGaN/GaN QWs was predicted early by theory and also evidenced experimentally ${ }^{13,14}$. The effectiveness of band profile engineering has recently been shown for staggered InGaN/GaN QW where compositional gradients of QWs have been achieved during the growth ${ }^{15}$. A linearly increasing InN composition in thick QWs furthermore showed efficient suppression of Auger recombination ${ }^{16}$. However, compositional grading on a nanometer scale is difficult to realize during epitaxial growth and very versatile profiles may be achieved by a combination of staggered growth and QWI. Implantation enhanced QWI was recently applied to AlGaN/AlN superlattices for spatially defined control of absorption properties ${ }^{17}$.

In this paper we will review our recent attempts in achieving QWI in InGaN/GaN MQW starting with a description of implantation damage build-up ${ }^{18}$ followed by the discussion of the effects induced by annealing of the as-grown and implanted structures ${ }^{19,20}$.

\section{EXPERIMENTAL DETAILS}

Two $\operatorname{In}_{x} \mathrm{Ga}_{1-\mathrm{x}} \mathrm{N} / \mathrm{GaN}$ MQW samples were grown by Metal-Organic Chemical Vapor Deposition (MOCVD) on a (0001) GaN/sapphire template at Rensselaer Polytechnic Institute, New York, USA. Typical growth conditions of these samples have been described elsewhere ${ }^{21,22}$. The first sample, RPI203, was used to study the implantation damage build-up upon nitrogen implantation (section 3.1) and consists of $5 \mathrm{In}_{\mathrm{x}} \mathrm{Ga}_{1-\mathrm{x}} \mathrm{N} / \mathrm{GaN}$ periods. The second sample, RPI209, consists of 10 $\mathrm{In}_{\mathrm{x}} \mathrm{Ga}_{1-\mathrm{x}} \mathrm{N} / \mathrm{GaN}$ periods and was used for annealing and QWI studies (section 3.2). The QWs have a thickness of $3 \mathrm{~nm}$ and an average InN molar fraction of $\mathrm{x}=10 \%$ (determined by Rutherford Backscattering Spectrometry, RBS). The QWs are separated by $20 \mathrm{~nm}$ thick GaN spacer layers. Finally, a $\sim 20 \mathrm{~nm} \mathrm{GaN}$ capping layer was deposited to protect the MQW region. Photoluminescence (PL) and ionoluminescence measurements reveal green QW emission despite the relatively low InN content, attributed to compositional fluctuations as evidenced by transmission electron microscopy (TEM) on a similar sample of the same sample set ${ }^{23}$.

Ion implantation and in-situ Rutherford Backscattering Spectrometry in channeling mode (RBS/C) were carried out on sample RPI203 in the double-beam chamber at the University of Jena, Germany ${ }^{18}$. The implantations were performed at 
room temperature (RT) with $35 \mathrm{keV} \mathrm{N}^{+}$ions. At this energy the maximum of the damage profile is placed in the central region of the MQWs. According to Monte Carlo simulations with the SRIM code ${ }^{24}$ the mean $\mathrm{N}$ range is $55 \mathrm{~nm}$, with a longitudinal straggling of $25 \mathrm{~nm}$. The ion fluence was increased sequentially from $5 \times 10^{12}$ up to $4 \times 10^{16} \mathrm{~cm}^{-2}$ and the sample was tilted by $7^{\circ}$ off-axis during the implantation to avoid channeling effects. The damage accumulation was monitored after each implantation step acquiring in-situ RBS/C spectra along the $<0001>$ aligned direction using a $2.2 \mathrm{MeV} \mathrm{He}{ }^{+}$beam and a 3-axis goniometer for sample orientation ${ }^{18}$. RBS/C allows a depth resolved assessment of crystal quality ${ }^{25}$.

Sample RPI209 was cut into 4 pieces. Two of the pieces were implanted at RT with nitrogen using three different energies of 35, 80 and $160 \mathrm{keV}$ to a total fluence of $7 \times 10^{13} \mathrm{~cm}^{-2}{ }^{19}$ These implantation conditions yield a homogeneous defect profile throughout the MQW region with low defect levels. One as-implanted sample was kept as a reference. An isochronous annealing program was performed on one implanted and one as-grown piece of sample RPI209. Rapid thermal annealing in flowing nitrogen up to $1000^{\circ} \mathrm{C}$ did not yield any changes in PL or X-Ray Diffraction (XRD) studies. Higher annealing temperatures at atmospheric pressure lead to the dissociation of $\mathrm{GaN}^{26}$. Therefore further annealing was performed using high temperature and high pressure (HTHP) at $1250{ }^{\circ} \mathrm{C}$ and $1400{ }^{\circ} \mathrm{C}$ in a $1.1 \mathrm{GPa}_{2}$ atmosphere for $30 \mathrm{~min}$. The HTHP annealing was performed at UNIPRESS, Poland and suppresses the dissociation of $\mathrm{GaN}$ at temperatures well above the MOCVD growth temperature ${ }^{27,28}$.

Ex-situ analysis of these samples was performed using XRD and PL. XRD measurements were performed on a D8Discover high resolution diffractometer (Bruker-AXS) using $\mathrm{Cu}\left(\mathrm{K}_{\alpha 1}\right)$ radiation equipped with a Göbel mirror, an asymmetric two-bounce $\mathrm{Ge}(220)$ monochromator and a NaI scintillation detector. To reduce the divergence, the primary $\mathrm{X}$-ray beam is collimated with a $0.2 \mathrm{~mm}$ slit in front of the monochromator and the secondary beam is collimated with a $0.1 \mathrm{~mm}$ slit placed at the detector entrance. Steady state PL spectroscopy was performed as a function of temperature (from $14 \mathrm{~K}$ to RT) using a cold finger He cryostat. The $325 \mathrm{~nm}$ line of a cw He-Cd laser (power density $\mathrm{I}_{0}<0.6 \mathrm{~W} . \mathrm{cm}^{-2}$ ) was used as excitation source. The sample luminescence was dispersed by a SPEX 1704 monochromator (1 m, 1200 gr.mm ${ }^{-1}$ ) and detected by a cooled Hamamatsu R928 photomultiplier ${ }^{19}$.

\section{RESULTS AND DISCUSSION}

\subsection{Implantation damage build-up}

Figure 2a shows the in-situ RBS/C spectra for $35 \mathrm{keV} \mathrm{N}$ implantation at RT. The low minimum yield of the as-grown sample, $\chi_{\min }=4.4(1) \%$ for window $\mathrm{W}_{1}$ and $2.2(1) \%$ for window $\mathrm{W}_{2}$ i.e. for $\mathrm{In}\left(\mathrm{W}_{1}\right)$ and $\left.\mathrm{Ga}_{(\mathrm{W}}\right)$, respectively, reveals the excellent single crystalline quality of the QWs and spacer layers. The minimum yield is defined as the ratio of the backscattering yield in the aligned spectrum to that of the random spectrum within a region of interest. Implantation will displace atoms from their lattice sites and the minimum yield is expected to increase with increasing fluence since the defects that are located within the free space between atomic rows lead to an increase of the backscattering yield in the aligned spectra. As expected, the RBS/C spectra show a successive augmentation of the backscattering yield in the aligned spectra with increasing ion fluence. Below a fluence of $\sim 1 \times 10^{15} \mathrm{~cm}^{-2}$ the damage level remains very low, only visible in the RBS/C spectra in a logarithmic scale. For higher fluences the damage level increases more rapidly. For a more quantitative analysis of the damage build-up the difference in the minimum yield of the implanted and virgin sample, $\Delta \chi_{\min }=\chi_{\min }$ (implanted)- $\chi_{\min }$ (virgin) was determined and plotted as a function of the fluence in Fig. $2 \mathrm{~b}$. The sigmoidal shaped damage build-up curve is typical for ion implantation in $\mathrm{GaN}$ and InGaN and reflects a high degree of dynamic annealing, i.e. due to effective point defect migration and recombination during the implantation, damage buildup proceeds very slowly in some fluence regions ${ }^{29-32}$. The experimental data are well described by fits (lines in Fig. 2b) using the Hecking model of damage build-up ${ }^{33}$ where the curve can be divided into four regimes as indicated in Fig. $2 \mathrm{~b}$ for the Ga signal. For low fluences in regime I, the damage build-up increases approximately linearly with the implantation fluence. When displacement cascades start to overlap, in regime II, an increased recombination of point defects leads to a slow down or saturation of damage accumulation. The strong increase of lattice damage in regime III is associated with the formation of stable extended defects such as clusters and stacking faults. For the highest fluences (regime IV) again a saturation of the maximum defect level is seen, while the defect profile is broadening and extending into deeper layers (see Fig. 2a) before eventually the aligned spectrum is reaching the random level. This complete loss of the channeling effect is often related with amorphization. In GaN, however, it is known that this regime is typical for the formation of a nanocrystalline phase rather than amorphization ${ }^{34,35}$. 

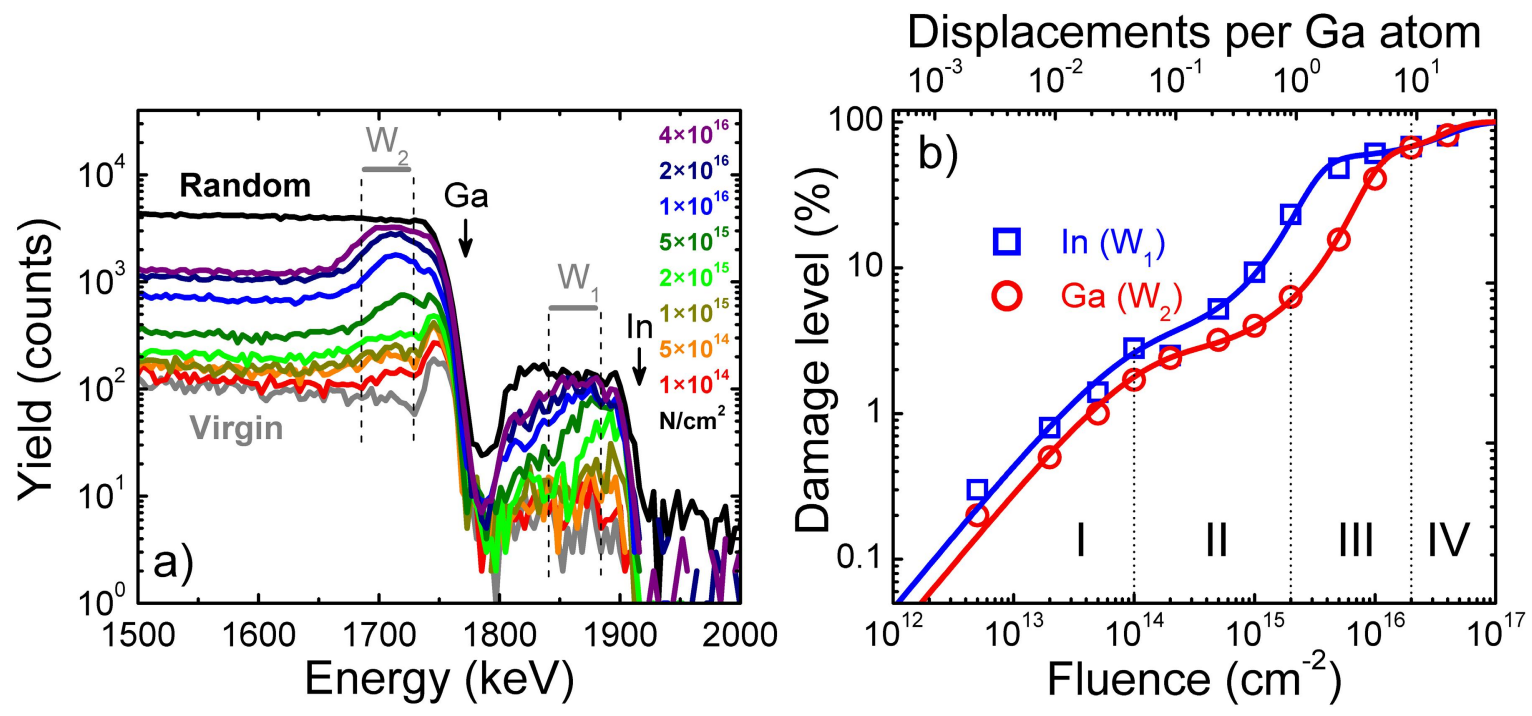

Figure 2. a) In-situ RBS/C spectra of sample RPI203 implanted to different fluences of $35 \mathrm{keV} \mathrm{N}$ ions. b) The damage level determined within the implanted layer (within windows $\mathrm{W}_{1}$ and $\mathrm{W}_{2}$ marked in a)). Solid lines represent fits to the data using Hecking's model (for details see text). (Adapted from ref. [18])

The different regimes described above are indicated in Fig. $2 b$ for the case of the curve for Ga. The curve for In shows a qualitatively similar behavior, however, the transitions for regime III and IV occur for slightly lower fluences and, in contrast to $\mathrm{GaN}$, for the highest fluence the random level is reached. This shows that InGaN is less radiation resistant than $\mathrm{GaN}$ in agreement with previous studies ${ }^{31,36}$. Note that the signal from $\mathrm{Ga}$ is averaged over the QWs and spacer layers thus mainly arising from the GaN spacer layers. Previous work comparing RBS/C data with TEM images suggested that regime I is mainly governed by the production of point defects while the density of extended defects such as clusters, dislocation loops and stacking faults is $\operatorname{low}^{34,37,38}$. For higher fluences, however, the density of extended defects increases strongly. Since these extended defects, in particular stacking faults, were shown to be thermally very stable ${ }^{39}$, we chose to implant a low fluence of $7 \times 10^{13} \mathrm{~cm}^{-2}$, corresponding to regime I, for subsequent QWI studies. For such low implantation fluences and defect levels, XRD was shown to be more sensitive to implantation defects than $\mathrm{RBS} / \mathrm{C}$ since point defects introduce high hydrostatic strain leading to an expansion of the $c$-lattice constant of the wurtzite nitride lattice ${ }^{40,41}$.

\subsection{Annealing and QWI studies}

Fig. 3 shows XRD 20- $\omega$ scans around the 0002 reflection of the as-grown sample RPI209 as well as of annealed, asimplanted and implanted+annealed pieces of this sample. The as-grown sample shows a high number of superlattice (SL) peaks, up to seventh order, revealing high quality interfaces of the MQW. HTHP annealing at $1250^{\circ} \mathrm{C}$ does not induce any significant changes neither in the XRD scans nor in the optical properties (not shown). After HTHP annealing at $1400{ }^{\circ} \mathrm{C}$ a significant decrease of the SL peak intensity is observed which can be due to intermixing but also due to the deterioration of the crystal quality such as the occurrence of phase separation. The as-implanted sample continues to show intense SL peaks confirming little effect of the implantation on the interface properties, however, all SL peaks are seen to shift to lower angles in agreement with previous implantation studies in SL samples ${ }^{42}$ and attributed to the expansion of the $c$-lattice parameter typically observed in ion implanted III-nitrides due to hydrostatic strain introduced by implantation defects ${ }^{38,41,43}$. Very high implantation fluences, on the other hand, can lead to ballistic intermixing and a strong decrease of SL peaks ${ }^{44}$. After HTHP annealing of this sample at $1400{ }^{\circ} \mathrm{C}$, the SL peaks are seen to shift back towards their original positions but their intensity is significantly decreased. This result indeed shows a weakening of the lattice after implantation as desired for QWI, i.e. the breaking of the bonds leads to enhanced mobility of the atoms during annealing. However, as will be discussed below the implantation/annealing treatment does not lead to the desired blue shift of the QW emission due to intermixing, but to a strong deterioration of the sample's optical properties (Fig. 4). 


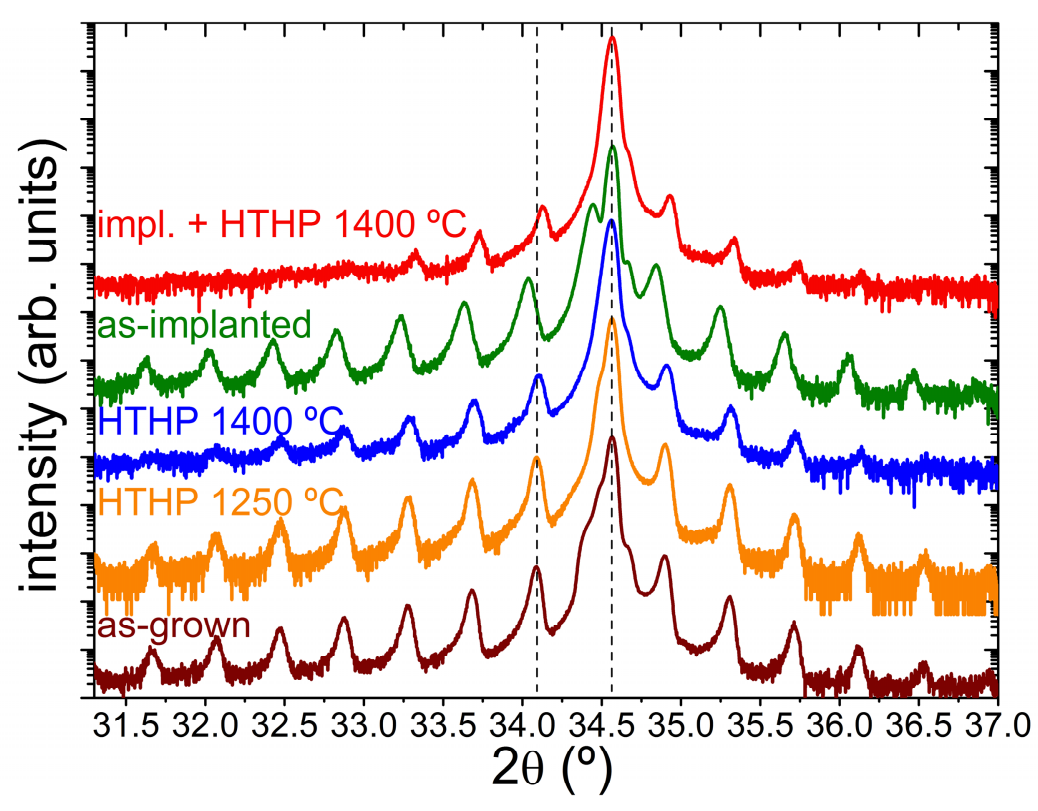

Figure 3. XRD 20- $\omega$ curves of the 0002 reflection for the RPI209 samples: as-grown, HTHP annealed at 1250 and $1400{ }^{\circ} \mathrm{C}$, as-implanted and implanted plus HTHP annealed at $1400^{\circ} \mathrm{C}$.

Figure 4 presents the RT and low temperature PL spectra of the as-grown, as-implanted and HTHP $1400{ }^{\circ} \mathrm{C}$ annealed samples ${ }^{19}$. The inset in Fig. $4 \mathrm{~b}$ shows a photograph of the bright green PL of the as-grown sample at low temperature. This green luminescence band (GB) is the dominant emission of this sample at both measuring temperatures. The width of this QW emission band is relatively broad (FWHM $\sim 180 \mathrm{meV}$ ), as frequently seen for InGaN QWs ${ }^{45}$, attributed to compositional and well width fluctuations evidenced by $\mathrm{TEM}^{23}$. Time resolved and temperature dependent PL data, in addition to the typical line broadening, indicate that the green QW emission is due to localized exciton recombination ${ }^{19}$. After implantation the GB is strongly quenched due to the introduction of non-radiative recombination pathways via implantation defects; the GB is not recovered after annealing. Annealing the as-grown sample leads to a high energy shift (by roughly $100 \mathrm{meV}$ ) of the GB consistent with QWI as also indicated by the XRD results. However, another broad band in the blue spectral region (BB) is also induced by the annealing treatment. Detailed PL analysis suggests that it is also due to excitonic transitions ${ }^{19}$. This bimodal luminescence indicates that annealing increases the spatial compositional fluctuations within the QWs leading to regions with considerably lower $\mathrm{InN}$ concentration. This results is in contrast to annealing studies of quantum dot samples which revealed a shrinkage of the quantum dots and homogenization of the InGaN quantum dots and wetting layers ${ }^{46}$. Another annealing report evidenced laterally inhomogeneous luminescence after annealing of InGaN LEDs, however, setting in at significantly lower temperatures $\left(950{ }^{\circ} \mathrm{C}\right)$ and accompanied by a strong decrease of the emission intensity ${ }^{47}$. Lateral luminescence inhomogeneities were also reported for InGaN laser structures where thermal decomposition took place during the high temperature growth of the p-type layer ${ }^{48}$. In this study, TEM analysis revealed the decomposition of the InGaN QWs into metallic In clusters and adjacent regions with lower InN contents in the QW.

\section{CONCLUSIONS}

The investigated InGaN/GaN QWs show exceptional thermal stability. No significant changes in structural and optical properties were observed up to an annealing temperature of $1250^{\circ} \mathrm{C}$. In contrast, earlier results for annealing at low nitrogen pressures showed strong deterioration already at significantly lower temperatures ${ }^{47,48}$. However, it should be noted that similar annealing conditions as those used in this study were used for InGaN QW based LED structures and evidenced the loss of QW emission already at $1100^{\circ} \mathrm{C}^{20}$ suggesting that sample structure and native defects play an important role in the sample's response to thermal treatment. 


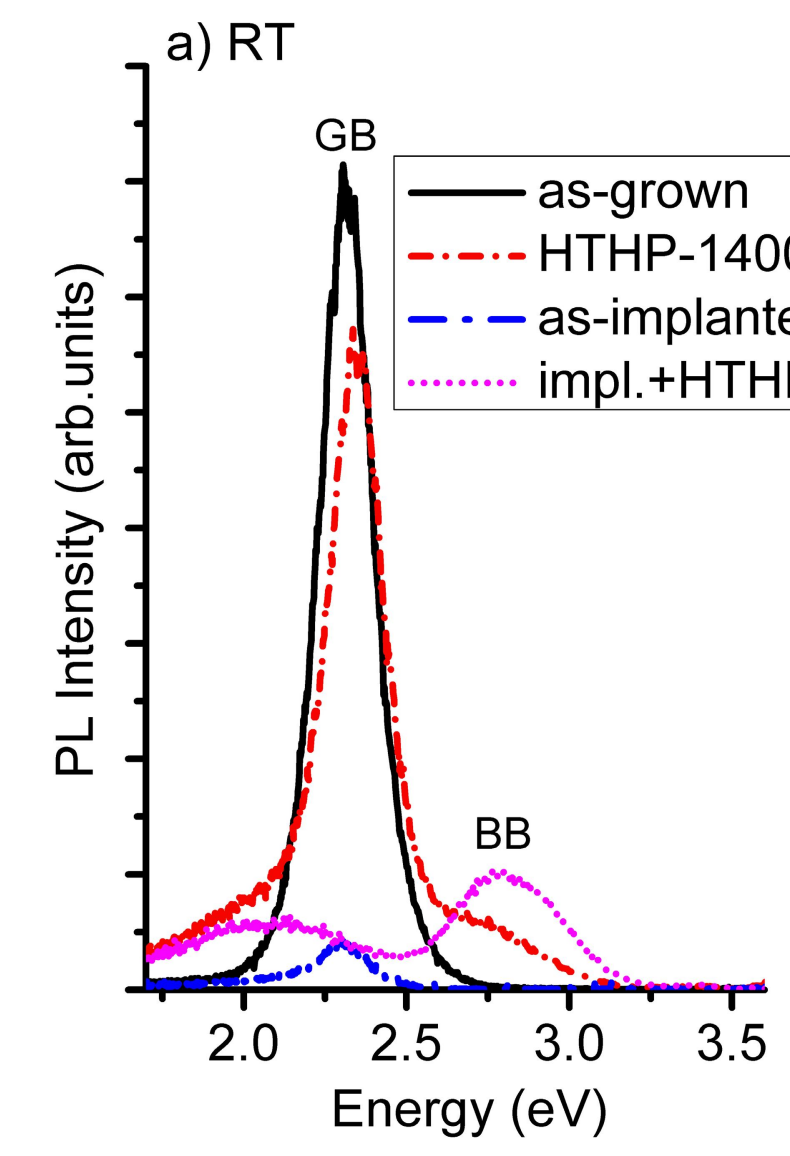

b) $T=14 \mathrm{~K}$

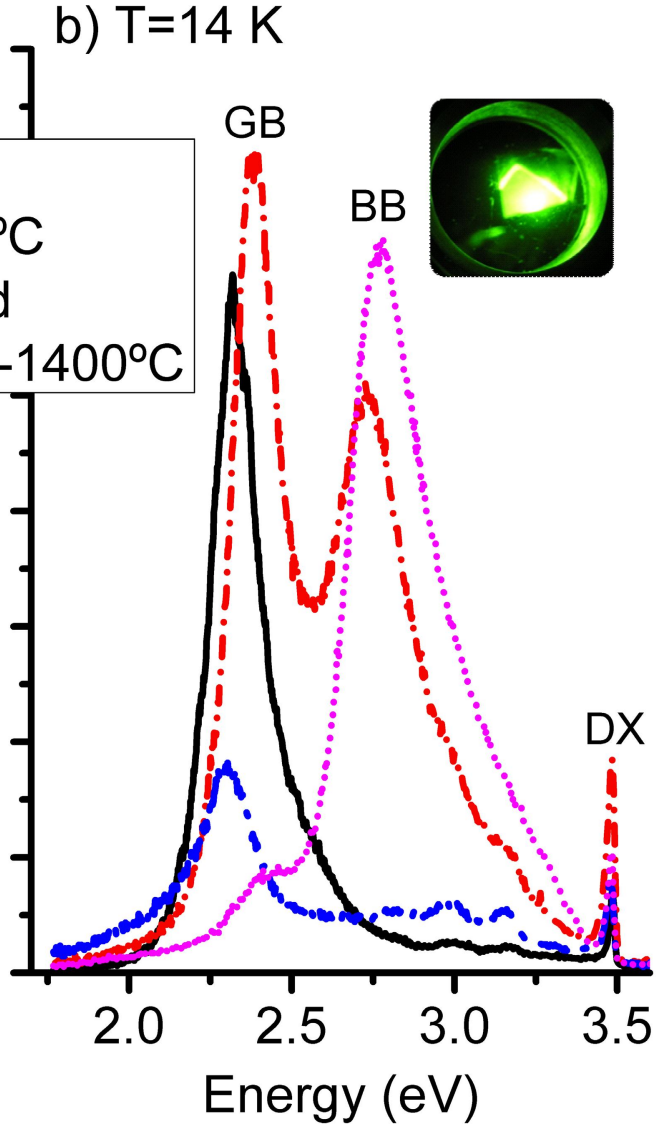

Figure 4. PL spectra of the as-grown and as-implanted sample RPI209 before and after HTHP annealing at $1400{ }^{\circ} \mathrm{C}$ measured at a) RT and b) $14 \mathrm{~K}$. The in-set shows a photograph of the low temperature PL of the as-grown sample. (Adapted from ref. [19])

For example the enhancement of In-Ga interdiffusion in the presence of Mg was suggested by McCluskey et al. who observed similar effects of HTHP annealing as presented here but starting at slightly lower temperatures ${ }^{14}$. Such discrepancies between the responses of different $\mathrm{InGaN} / \mathrm{GaN}$ QWs to annealing are complicating the optimization of annealing parameters for QWI. For annealing at $1400^{\circ} \mathrm{C}$ bimodal emission suggests increased spatial compositional fluctuations. Implantation enhanced QWI was found to be challenging due to the severe effect of implantation defects on the QWs' optical properties. In fact, for the chosen conditions the QW luminescence was completely quenched and could not be recovered during thermal annealing. Lower implantation fluences or the use of swift heavy ions may be useful to keep implantation damage low. It was also reported recently that area-selective intermixing is possible by using metal/dielectric-coatings where metals such as Mo were shown to enhance interdiffusion during thermal annealing ${ }^{49}$.

\section{ACKNOWLEDGEMENTS}

We thank C. Wetzel, Rensselaer Polytechnic Institute, USA, for the InGaN QW samples; M. Boćkowski, Unipress, Poland for the HTHP annealing and E. Wendler, University of Jena, Germany for the help in performing the in-situ RBS/C measurements and D. Carvalho, T. Ben, F.M. Morales, University of Cadiz, Spain for TEM analysis. We acknowledge funding by FCT Portugal (PTDC/FIS-NAN/0973/2012, UID/CTM/50025/2013, and RECI/FISNAN/0183/2012 (FCOMP-01-0124-FEDER-027494 and individual grants SFRH/BD/111733/2015 (MCS), SFRH/BPD/111285/2015 (MP), SFRH/BD/76300/2011 (JR), and Investigador FCT (KL)). ARC acknowledges Juan de la Cierva grant (under contract number JCI-2012-14509, Spain. 


\section{REFERENCES}

[1] Damilano, B. and Gil, B., "Yellow-red emission from (Ga,In)N heterostructures," J. Phys. D: Appl. Phys. 48, 403001 (2015).

[2] Cho, J., Schubert, E. F., Kim, J.K., "Efficiency droop in light-emitting diodes: challenges and countermeasures," Laser Phot. Rev. 7, 408-421 (2013).

[3] Crawford, M. H., "LEDs for Solid-State Lighting: Performance Challenges and Recent Advances," IEEE J. Sel. Topics in Quantum Electr. 15, 1028-1040 (2009).

[4] Wang, X., Ren, X., Kahen, K., Hahn, M. A., Rajeswaran, M., Maccagnano-Zacher, S., Silcox, J., Cragg, G. E., Efros, A. L., Krauss, T. D., "Non-blinking semiconductor nanocrystals," Nature 459, 686-689 (2009).

[5] O’Donnell, K. P., Auf der Maur, M., Di Carlo, A., Lorenz, K., "It's not easy being green: Strategies for allnitrides, all-colour solid state lighting," PSS RRL 6, 49-52 (2012).

[6] Auf der Maur, M., Lorenz, K., Di Carlo, A., "Band gap engineering approaches to increase InGaN/GaN LED efficiency," Opt Quant Electron 44, 83-88 (2012).

[7] Deppe, D.G., and Holonyak, N., "Atom diffusion and impurity induced layer disordering In quantum well III-V semiconductor heterostructures," J. Appl. Phys. 64, R93-R113 (1988).

[8] Beal, R., Aimez, V., and Dubowski, J. J., "Excimer laser induced quantum well intermixing: a reproducibility study of the process for fabrication of photonic integrated devices ," Optics Express 23, 1073-1080 (2015).

[9] Du, S. C., Fu, L., Tan, H. H., and Jagadish, C., "Investigation of ion implantation induced intermixing in InP based quaternary quantum wells," J. Phys. D Appl. Phys. 44, 475105 (2011).

[10] Shtepliuk, I., Khranovskyy, V., Yakimova R., "Effect of Zn-Cd interdiffusion on the band structure and spontaneous emission of $\mathrm{ZnO} / \mathrm{Zn} 1-\mathrm{xCdxO} / \mathrm{ZnO}$ quantum wells," Superlattices and Microstructures 85, 438-444 (2015).

[11] Coleman, V. A., Buda, M., Tan, H. H., Jagadish, C., Phillips, M. R., Koike, K., Sasa, S., Inoue M., and Yano, M., "Observation of blue shifts in $\mathrm{ZnO} / \mathrm{ZnMgO}$ multiple quantum well structures by ion-implantation induced intermixing," Semicond. Sci. Technol. 21, L25-L28 (2006).

[12] Davis, J. A., Dao, L. V., Wen, X., Ticknor, C., Hannaford, P., Coleman, V. A., Tan, H. H., Jagadish, C., Koike, K., Sasa, S., Inoue, M., and Yano, M., "Suppression of the internal electric field effects in $\mathrm{ZnO} / \mathrm{Zn0} 0.7 \mathrm{Mg} 0.3 \mathrm{O}$ quantum wells by ion-implantation induced intermixing," Nanotechnology 19, 055205 (2008).

[13] Chen, C. C., Hsueh, T. H., Ting, Y. S., Chi, G. C., Chang, C. A., ” Effects of In and Ga interdiffusion on the optical gain of InGaN/GaN quantum well,” J. Appl. Phys. 90, 5180 (2001).

[14] McCluskey, M.D., Romano, L.T., Krusor, B.S., Hofstetter, D., Bour, D.P., Kneissl, M., Johnson, N.M., Suski, T., Jun, J., "Disordering of InGaN/GaN superlattices after high-pressure annealing," MRS Internet J. Nitride Semicond. Res. 4S1, G3.42 (1999).

[15] Zhao, H.P., Liu, G.Y., Li, X. H., Arif, R.A., Huang, G.S., Poplawsky, J.D., Tafon Penn S., Dierolf, V., Tansu, N., " Design and characteristics of staggered InGaN quantum-well light-emitting diodes in the green spectral regime," IET Optoelectron. 3, 283-295 (2009).

[16]Zhang, Z.H., Liu, W., Ju,Z., Tan, S. T., Ji, Y., Kyaw, Z., Zhang, X., Wang, L., Sun, X. W., and Demir, H. V., ” InGaN/GaN multiple-quantum-well light-emitting diodes with a grading InN composition suppressing the Auger recombination," Appl. Phys. Lett. 105, 033506 (2014).

[17] Wierer, J. J., Allerman, A. A., Skogen, E. J., Tauke-Pedretti, A., Alford, C., Vawter, G. A., and Montaño, I., "Layer disordering and doping compensation of an intersubband AlGaN/AlN superlattice by silicon implantation," Appl. Phys. Lett. 105, 131107 (2014).

[18] Redondo-Cubero, A., Lorenz, K., Wendler, E., Magalhães, S., Alves, E., Carvalho, D., Ben, T., Morales, F. M., García, R., O’Donnell, K. P., and Wetzel, C., "Analysis of the stability of InGaN/GaN multiquantum wells against ion beam intermixing," Nanotechnology 26, 425703 (2015).

[19] Sousa, M.A., Esteves, T.C., Ben Sedrine, N., Rodrigues, J., Lourenço, M.B., Redondo-Cubero, A., Alves, E., O’Donnell, K.P., Bockowski, M., Wetzel, C., Correia, M.R., Lorenz, K., Monteiro, T., "Influence of nitrogen implantation and thermal annealing on the optical properties of green emitting InGaN/GaN multiple quantum wells," Scientific Reports 5, 09703 (2015).

[20] Ben Sedrine, N., Esteves, T. C., Rodrigues, J., Rino, L., Correia, M. R., Sequeira, M. C., Neves, A. J., Alves, E., Bockowski, M., Edwards, P. R., O'Donnell, K.P., Lorenz K., and Monteiro, T., "Photoluminescence studies of a perceived white light emission from a monolithic InGaN/GaN quantum well structure," Scientific Reports 5, 13739 (2015). 
[21] Wetzel, C., Salagaj, T., Detchprohm, T., Li, P., and Nelson, J. S., "GaInN / GaN growth optimization for highpower green light-emitting diodes," Appl. Phys. Lett. 85, 866-868 (2004).

[22] Detchprohm, T., Zhu, M., Xia, Y., Li, Y., Zhao, W., Senawiratne, J. \& Wetzel, C., "Improved performance of GaInN based deep green light emitting diodes through V-defect reduction," Physica Status Solidi c 5, 22072209 (2008).

[23] Carvalho, D., Morales, F. M., Ben, T., García, R., Redondo-Cubero, A., Alves, E., Lorenz, K., Edwards, P. R., O’Donnell, K. P., and Wetzel, C., "Quantitative Chemical Mapping of InGaN Quantum Wells from Calibrated High-Angle Annular Dark Field Micrographs," Microsc. Microanal. 21, 994-1005 (2015).

[24]Ziegler, J. F., Biersack, J.P., Littmark, U., [The stopping and range of ions in solids], Pergamon Press, New York, 1 (1985).

[25] Redondo-Cubero, A. , Lorenz, K., Gago, R., Franco, N., di Forte Poisson, M.A., Alves, E., and Muñoz, E., "Depth-resolved analysis of spontaneous phase separation in the growth of lattice-matched AlInN" J. Phys. D: Appl. Phys. 43, 055406 (2010).

[26] Bartels, J., Freitag, K., Marques, J. G., Soares, J. C., and Vianden, R., "Incorporation of the transition metal Hf into GaN," Hyperfine Interactions 120-121, 397 (1999).

[27] Roqan, I. S., O’Donnell, K. P., Martin, R. W., Edwards, P. R., Song, S. F., Vantomme, A., Lorenz, K., Alves, E., and Boćkowski, M., "Identification of the prime optical center in GaN:Eu3+," Phys. Rev. B 81, 085209 (2010).

[28] Lorenz, K., Miranda, S. M. C., Alves, E., Roqan, I. S., O’Donnell, K. P., and Boćkowski, M., "High pressure annealing of Europium implanted GaN," Proc. SPIE 8262, 82620C (2012).

[29] Kucheyev, S. O., Williams, J. S., Jagadish, C., "Ion-beam-defect processes in group-III nitrides and ZnO," Vacuum 73, 93-104 (2004).

[30] Lorenz, K., Peres, M., Franco, N., Marques, J. G., Miranda, S. M. C., Magalhães, S., Monteiro, T., Wesch, W., Alves, E., Wendler, E., "Radiation damage formation and annealing in $\mathrm{GaN}$ and $\mathrm{ZnO,"} \mathrm{Proc.} \mathrm{SPIE} \mathrm{7940,}$ 79400 O (2011).

[31] Kucheyev, S. O., Williams, J.S., Zou, J., and Jagadish, C., "Dynamic annealing in III-nitrides under ion bombardment," J. Appl. Phys. 95, 3048 (2004).

[32] Wendler, E., Kamarou, A., Alves, E., Gärtner, K., Wesch, W., "Three-step amorphisation process in ionimplanted GaN at 15 K," Nucl. Instrum. Meth. Phys. Res. B 206, 1028-1032 (2003).

[33] Hecking, N., Heidemann, K. F., and TeKaat, E., "Model of temperature dependent defect interaction and amorphization in crystalline silicon during ion irradiation," Nucl. Instrum. Meth. Phys. Res. B 15, 760-764 (1986).

[34] Gloux, F., Wojtowicz, T., Ruterana, P., Lorenz, K., Alves, E., "Investigation of the crystallographic damage formed in GaN by medium range energy rare earth ion implantation," J. Appl. Phys 100, 073520 (2006).

[35] Ruterana, P., Lacroix, B., and Lorenz, K., "A mechanism for damage formation in GaN during rare earth ion implantation at medium range energy and room temperature," J. Appl. Phys. 109, 013506 (2011).

[36] Wendler, E., Wesch, W., Alves, E., Kamarou A.," Comparative study of radiation damage in GaN and InGaN by $400 \mathrm{keV}$ Au implantation," Nucl. Instr. and Meth. B 218, 36-41 (2004).

[37] Lorenz, K., Barradas, N. P., Alves, E., Roqan, I. S., Nogales, E., Martin, R. W., O'Donnell, K. P., Gloux, F., Ruterana, P., "Structural and optical characterization of Eu-implanted GaN," J. Phys. D: Appl. Phys. 42, 165103 (2009).

[38] Turos, A., "On the mechanism of damage buildup in gallium nitride," Radiation Effects \& Defects in Solids $168,431-441$ (2013).

[39] Wojtowicz, T., Gloux, F., Ruterana, P., Lorenz, K., and Alves, E., “TEM investigation of Tm implanted GaN, the influence of high temperature annealing," Optical Materials 28, 738-741 (2006).

[40] Marques, J. G., Lorenz, K., Franco, N., Alves, E., “Defect production in neutron irradiated GaN,” Nucl. Instr. Meth. Phys. Res. B 249, 358-361 (2006).

[41] Lacroix, B., Leclerc, S., Declémy, A., Lorenz, K., Alves E., and Ruterana, P., "Mechanisms of damage formation in Eu-implanted GaN probed by X-ray diffraction," Europhysics Lett. 96, 46002 (2011).

[42] Magalhães, S., Peres, M., Fellmann, V., Daudin, B., Neves, A. J., Alves, E., Monteiro, T., and Lorenz, K., "Functionalizing self-assembled GaN quantum dot superlattices by Eu-implantation," J. Appl. Phys. 108, 084306 (2010).

[43] Liu, C., Mensching, B., Volz, K., Rauschenbach, B., "Lattice expansion of Ca and Ar ion implanted GaN," Appl. Phys. Lett. 71, 2313-2315 (1997). 
[44] Redondo-Cubero, A., Lorenz, K., Wendler, E., Carvalho, D., Ben, T., Morales, F.M., García, R., Fellmann, V., Daudin, B., "Selective ion-induced intermixing and damage in low-dimensional GaN/AlN quantum structures," Nanotechnology 24, 505717 (2013).

[45] Chen, J.H., Feng, Z.-C., Tsai, H.L., Yang, J.R., Li, P., Wetzel, C., Detchprohm, T., Nelson, J., "Optical and structural properties of InGaN/GaN multiple quantum well structure grown by metalorganic chemical vapor deposition," Thin Solid Films 498, 123-127 (2006).

[46] Wang, Q., Wang, T., Bai, J., Cullis, A. G., Parbrook, P. J., Ranalli, F., "Influence of annealing temperature on optical properties of InGaN quantum dot based light emitting diodes," Appl. Phys. Lett. 93, 081915 (2008).

[47] Jeong, T.S., Kim, J.H., Han, M.S., Lim, K.Y., Youn, C.J., "X-ray and cathodoluminescence study on the effect of intentional long time annealing of the InGaN/GaN multiple quantum wells grown by MOCVD," Journal of Crystal Growth 280, 357-363 (2005).

[48]Li, Z., Liu, J., Feng, M., Zhou, K., Zhang, S., Wang, H., Li, D., Zhang, L., Zhao, D., Jiang, D., Wang, H., and Yang, H., "Suppression of thermal degradation of InGaN/GaN quantum wells in green laser diode structures during the epitaxial growth,"Appl. Phys. Lett. 103, 152109 (2013).

[49] Shen, C., Ng, T. K., and Ooi, B. S., "Enabling area-selective potential-energy engineering in InGaN/GaN quantum wells by post-growth intermixing," Optics Express 23, 7991-7998 (2015). 